\title{
Botanische Exkursion nach Gran Canaria
}

\section{Mit dem AK-Phyto der Gesellschaft für Ganzheitliche Tiermedizin unterwegs}

Die schon zur Tradition gewordene botanische Exkursion unter Leitung von Prof. Matthias Melzig führte uns vom 26. März bis 5. April 2017 nach Gran Canaria. Unsere Erwartungen waren hoch, gilt doch Gran Canaria als botanisch und geologisch sehr abwechslungsreich und wird deshalb gerne als „Miniaturkontinent“ bezeichnet.

\section{Hinab in den Vulkan}

Der Landeanflug auf die Hauptstadt Las Palmas ließ uns erst einmal skeptisch werden. Ausgedehnte Foliengewächshäuser, nackter Fels, kahle Erde, durchfurcht von breiten Straßen, diese gesäumt von haushohen Werbetafeln, entlang der Küste ausgedehnte Hotelund Gewerbekomplexe - das wirkte wenig einladend. Doch nur zehn Autominuten entfernt, im Landesinneren, umfing uns eine üppige, z.T. subtropische Vegetation in einer kontrastreichen Vulkanlandschaft ( $\mathbf{A b} \mathbf{b} \mathbf{b} \mathbf{1})$. Schon beim ersten Ausflug in die dicht bewachsene Caldera de Bandama offenbarte sich uns der große Artenreichtum der Insel. Über schwarze Lava-Asche ging es in vielen Kehren in den Vulkankrater hinab. Verschiedene Ginster- und Lavendelarten säumten den Weg, reich blühende Exemplare der mannshohen, strauchartigen Blütenreichen Winde (Convolvulus floridus L.) und der Ahornblättrigen Strauchpappel (Malva canariensis M.F. Ray). Mastixstrauch (Pistacia lentiscus L.), Kletten-Krapp (Rubia peregrina L.) und der Strauchige Krapp (Rubia fruticosa Aiton) mit seinen ledrigen, spitz gezähnten Blättern sorgten für dichtes Buschwerk. Dazwischen wuchsen Scharlachroter Erdrauch (Fumaria coccinea R.T.

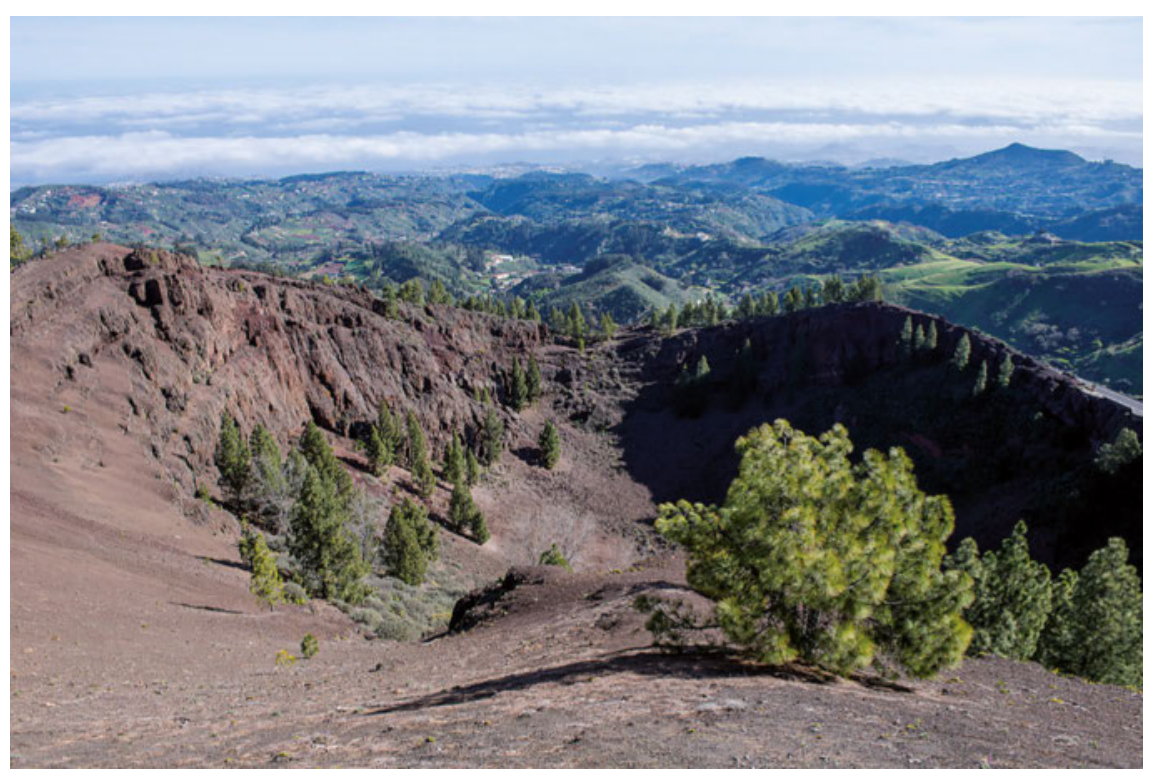

- Abb. 1 Das etwa 200 km westlich der südmarokkanischen Küste im Atlantik gelegene Gran Canaria gehört zu einem Archipel, der durch Vulkanismus entstanden ist. Es dauert seine Zeit, bis sich die Pflanzenwelt die vulkanischen Strukturen erobern kann. (c) Dr. Ferdinand Worm

Lowe ex Pugsley), Affodill (Asphodelus aestivus Brot.), ein reiches Spektrum an Schmetterlingsblütlern und verschiedene Arten von Gänsedisteln (Sonchus spp.). Diese erinnern in Blüten und Blättern an den Löwenzahn, erreichen jedoch eine Wuchshöhe von bis zu $2 \mathrm{~m}$. Stattliche Echium-Arten (Natternköpfe) prägten das Bild: Echium decaisnei Webb \& Berthel, Echium strictum L.f., Echium webbii Coincy, von Bienen und vielen anderen Insekten eifrig besucht. Am Grunde der Caldera bot uns ein knorriger Eukalyptus Schatten. Hier wachsen Kanarische Dattelpalmen (Phoenix canariensis Chabaud) und verschiedene YuccaArten sowie eine strauchige kanarische Unterart des Olivenbaums (Olea europaea ssp. cerasiformis G. Kunkel \& Sunding).

\section{Von importierten Läusen}

Auch dem Echten Feigenkaktus (Opuntia ficus barbarica A. Berger) begegneten wir hier - besiedelt von in früherer Zeit für die Kanaren wichtigen Parasiten, den Cochenille-Läusen ( $\triangleright$ Abb. 2). Aus ihnen gewann man in großem Stil „Cochenille“, Karminsäure zur Herstellung eines sehr begehrten roten Farbstoffs. Das war bis zur Entwicklung der synthetischen Azofarbstoffe auf Basis des Anilins durch die Badische Anilin- und Sodafabrik (BASF) ein durchaus lukratives Geschäft. In Lateinamerika war die Gewinnung von Cochenille schon seit vorchristlicher Zeit eine verbreitete Kulturtechnik. Mit dem besonders lichtechten Cochenille färbte man insbesondere herrschaftliche Gewänder und zahlte man seinen Tribut. 

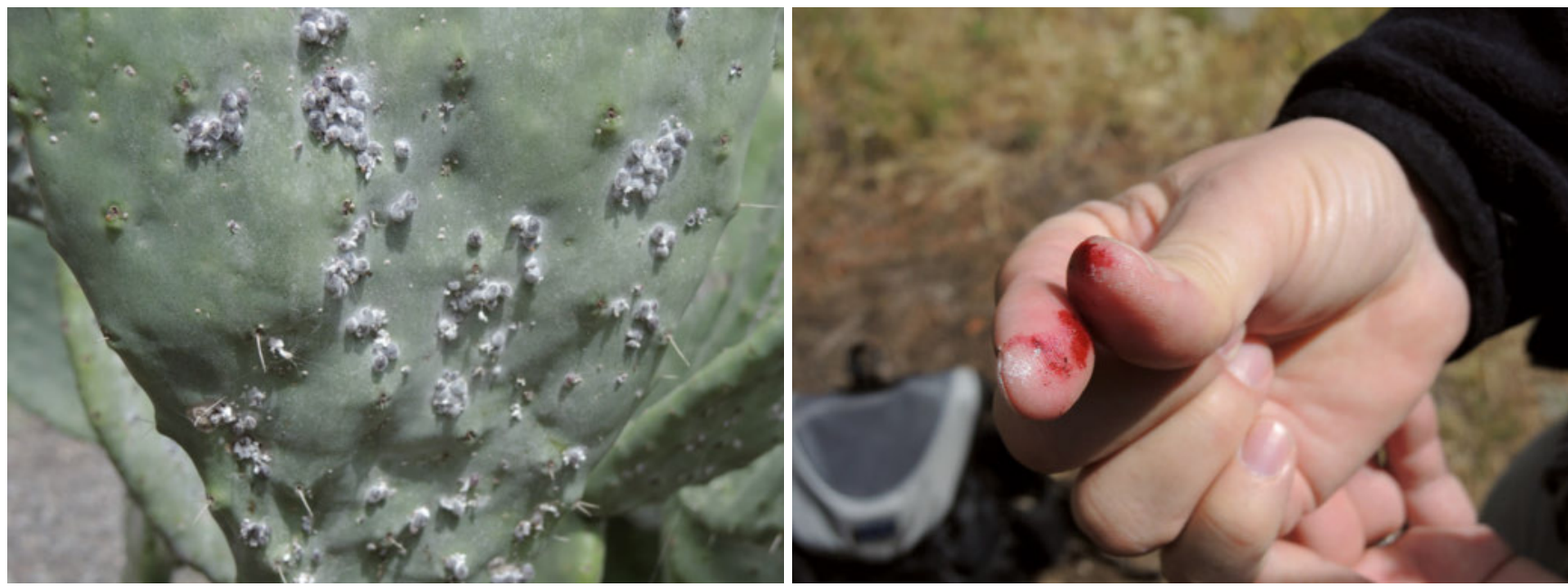

- Abb. 2100000 weibliche Cochenille-Schildläuse werden für die Herstellung von einem Kilo Farbstoff benötigt. Man trocknet die Läuse und kocht sie in Wasser unter Zusatz von Schwefelsäure. Mit Alaun und Kalk wird das Karmin ausgefällt und anschließend getrocknet. Aus einem kg Cochenille gewinnt man $50 \mathrm{~g}$ Karmin. Kein Wunder, dass man heute eher synthetische Farben verwendet. Unter dem Kürzel E120 ist Cochenille bis heute als Lebensmittelfarbstoff zugelassen. (c) Raimund Scherzer

Die Spanier, die als Kolonialherren in Südamerika das Monopol für das begehrte Cochenille hatten, holten die Opuntien samt ihrer Parasiten speziell für die Farbstoffgewinnung zu Beginn des 19. Jahrhunderts nach Gran Canaria und begannen dort und auf dem spanischen Festland erfolgreich, Cochenille-Läuse zu züchten. Dass es nicht immer gut geht, wenn man Pflanzen und Tiere aus fernen Biotopen holt, bewiesen übrigens die
Engländer. Bereits 1788 führten die ersten britischen Siedler Australiens die Kaktusfeige mitsamt Cochenille-Läusen in ihrer neuen Heimat ein. Vermutlich hatte es den Briten nicht gefallen, dass sie wegen des Cochenilles, das auch sie zum Färben herrschaftlicher Gewänder benötigten, von ihrem großen Konkurrenten zur See abhängig waren. So setzten sie ihre Hoffnung in eine eigene Produktion auf den riesigen Flächen Australiens. Und

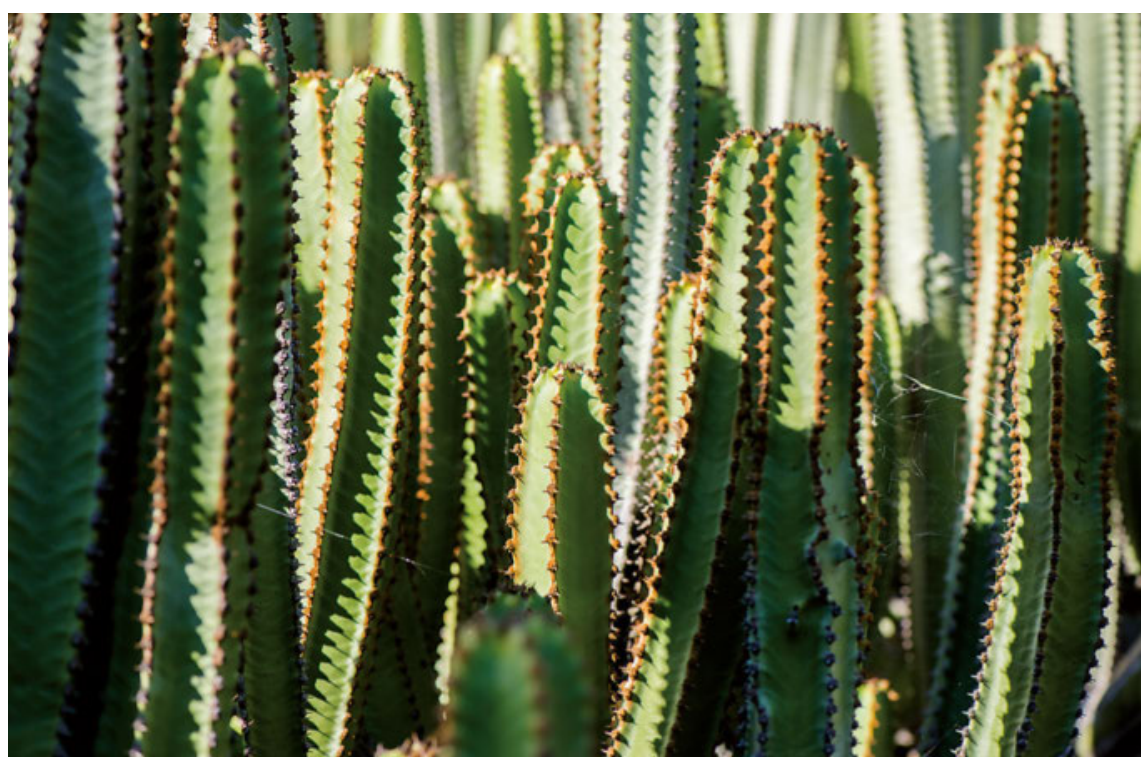

- Abb. 3 Die Kanaren-Wolfsmilch, Euphorbia canariensis, gedeiht besonders gut in der Vulkanasche erodierter Berghänge. Die sehr langsam wachsende Pflanze kann 100 Jahre alt werden. Sie lässt sich nur durch genaues Hinsehen von Kakteen unterscheiden. (c) Dr. Ferdinand Worm tatsächlich - die Kaktusfeigen gediehen prächtig, leider jedoch nicht die Cochenille-Läuse. Vom Traum des britischen Cochenilles australischer Herkunft blieb über 100 Jahre nur eine ausgedehnte Kaktusplage, der man erst durch Einführen eines weiteren südamerikanischen Kakteen-Schädlings Herr wurde.

\section{Heimat zahlreicher Euphorbiaceen}

Ihr für die kanarische Vegetation charakteristisches Aussehen verleihen der Caldera de Bandama die Wolfsmilchgewächse. Stattliche Exemplare der KönigJuba-Wolfsmilch (Euphorbia obtusifolia Lam.) sowie die Balsamwolfsmilch (Euphorbia balsamifera Aiton) prägen das Landschaftsbild. Die erodierten Steilhänge der Caldera bieten offenbar der Kanaren-Wolfsmilch (Euphorbia canariensis L.) ideale Lebensbedingungen. Hier gedeihen eindrucksvolle Exemplare dieser Art, die schon viele Jahrzehnte alt sein dürften. Sie lassen sich nur durch genaues Hinsehen von Kakteen unterscheiden ( $\bullet$ Abb. 3). Der den Pflanzen dieser Gattung eigene Milchsaft ist chemisch und pharmakologisch hoch interessant. Euphorbium, der an der Luft getrocknete Milchsaft, fand sich noch im ersten Deutschen Arzneibuch von 1871 
zur Herstellung blasenziehender Pflaster und scharfer Einreibungen. Heute wird Euphorbium aufgrund seiner extremen Schärfe und der heftigen Entzündungsauslösung selbst bei minimaler Dosis arzneilich nur noch in homöopathischen Formulierungen verwendet. Der Wirkstoff Resiniferatoxin des Euphorbiums ist 1000 mal schärfer als Capsaicin und dient als Ausgangssubstanz für die Entwicklung neuer Schmerzmittel. Die hochgradig entzündungsauslösend wirkenden Diterpenester des Milchsaftes können sowohl krebsauslösend als auch krebshemmend wirken. Am Rattenohr verursachen bereits 2,5 nl des Milchsaftes der Zypressenwolfsmilch (Euphorbia cyparissias L.) eine starke Entzündung. In der Volksheilkunde wurde die Milch der Garten-Wolfsmilch (Euphorbia peplus L.) z. B. lokal bei Warzen eingesetzt. Aufgrund der Gefahr einer Tumorentstehung ist dies nicht zu empfehlen.

Aus dieser Anwendungstradition entstand die Idee für ein modernes Medikament: Als isolierte Reinsubstanz sind Diterpenester aus Euphorbia peplus L. heute zur Behandlung von Hauterkrankungen wie der aktinischen Keratose, eine durch intensive Sonneneinstrahlung induzierte Präkanzerose, zugelassen (provozierte Entzündung mit Abtöten der Zellen). Proteasen aus EuphorbiaceenMilchsäften werden zudem zur Fibrinolyse und als Verdauungsenzyme eingesetzt. Die Industrie nutzt sie als antibakteriell und fungizid wirkende Waschmittelzusatzstoffe, zur Fleischreifung, Käseherstellung, zum Abbau von Gluten, Entfernen von Haaren bei der Lederherstellung u.v.m. Zudem enthalten EuphorbiaceenMilchsäfte reichlich Terpene, aus denen Biotreibstoff und Naturkautschuk gewonnen werden kann.

\section{Auf dem Wolkenfelsen}

Ein besonderes Erlebnis war der Besuch des 1813 Meter hohen Roque Nublo, der sich wie eine majestätische Skulptur über einem alten Kultplatz der Insel-Ureinwohner erhebt. Der „Wolkenfelsen“, der als Wahrzeichen Gran Canarias gilt, ist das Relikt eines erodierten Vulkans, der

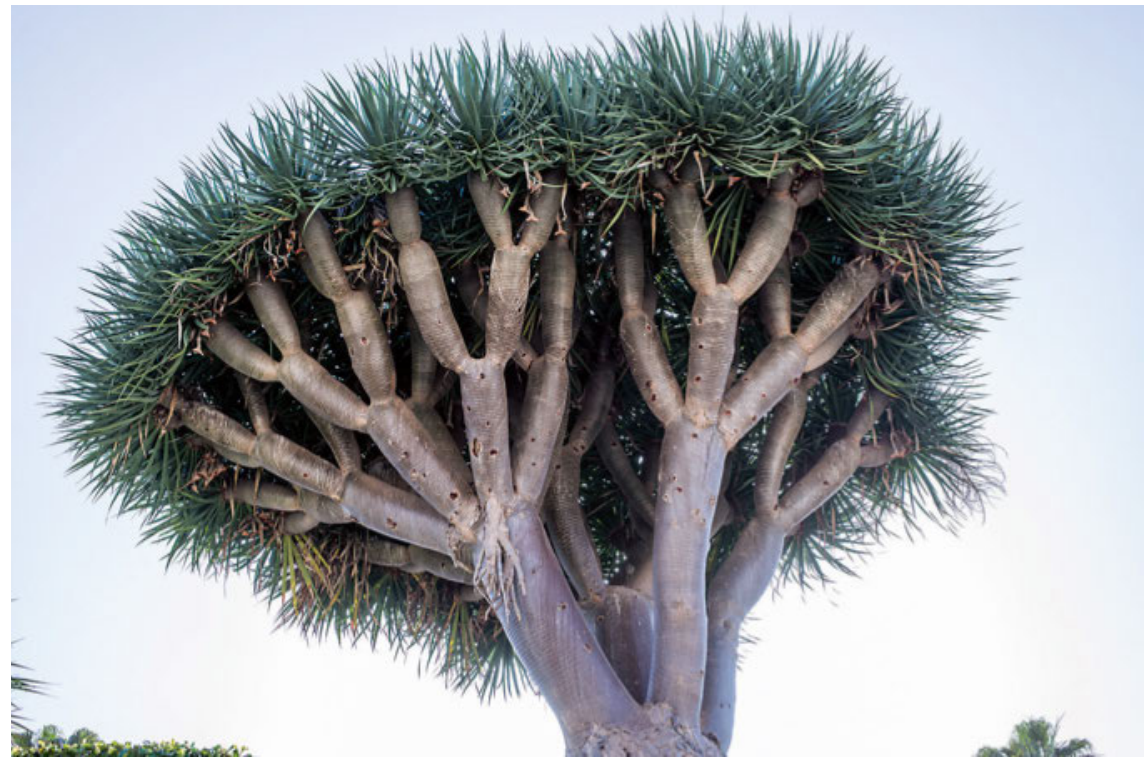

- Abb. 4 Drachenbäume (Dracaena draco) können mehrere hundert Jahre alt werden. Etwa 8-10 Jahre wachsen sie unverzweigt. Nach jeder Blühperiode, die sich etwa alle 15 Jahre wiederholt, kommt es zur wirteligen Verzweigung. Der Drachenbaum war einstmals ein typisches Gewächs der Kanaren, wurde aber zur Gewinnung von Venezianisch Rot stark dezimiert. @ Dr. Ferdinand Worm

heutigen Caldera de Tejeda. Von hier bot sich uns bei bester Fernsicht ein spektakulärer Blick über die gesamte Insel mit ihren tief eingegrabenen Barrancos, ihren Kratern und Hochebenen. In der Ferne war die Nachbarinsel Teneriffa zu sehen, mit ihrem höchsten Berg, dem Teide. Hier lernten wir den Kiefernwald der Insel kennen. Die Kanarenkiefer (Pinus canariensis C. Sm.) hat ihre nächste Verwandtschaft interessanterweise im Himalaya. Ihre langen, relativ weichen Nadeln, die zu dritt beieinander stehen, werden von den Landwirten vom Waldboden gesammelt und als Einstreu für Ziegen- und Schafställe genutzt. Hier wuchsen Andorn (Marrubium vulgare L.), das Wollige Gliedkraut (Sideritis dasygnaphala (Webb \& Berthels) Clos.) und die Baumwollstrohblume (Helichrysum gossypinum Webb), winzige Schönheiten wie das zu den Zistrosengewächsen gehörende gefleckte Sandröschen (Tuberaria guttata (L.) Fourr.) oder der Scheinkrokus (Romulea requienii Parl.).

\section{Hanglage: der Botanische Garten}

Eindrucksvoll war auch der Besuch des Botanischen Gartens von Gran Canaria. Er liegt am Steilhang des Barranco Guiniguada in Tafira und ist das Lebenswerk des schwedisch-spanischen Botanikers Erik Ragnar Svensson (1910-1973). Auf ca. 27 ha zeigt dieser Garten viele der fast 500 endemischen Pflanzenarten der Kanarischen Inseln sowie zahlreiche Pflanzen der übrigen makaronesischen Inseln (Azoren, Madeira, Kapverdische Inseln). Besonders beeindruckend ist die Sammlung der Euphorbien, die sich im Barranco offensichtlich wohlfühlen. Faszinierend auch die umfangreiche Sammlung der für die Kanaren typischen Vertreter aus der Familie der Dickblattgewächse, der Crassulaceae. Zu ihnen gehören auch die prächtigen Aeonien, die anspruchslos auf kargen Felsen wie auch auf ziegelgedeckten Hausdächern gedeihen und überall auf der Insel zu finden sind. Als Parkbaum gedeiht hier auch der Drachenbaum (Dracaena draco L.), einstmals ein typisches Gewächs der Kanaren ( Abb. 4). Das Harz der Rinde, Sanguis draconis, Kanarisches Drachenblut, enthält den begehrten Farbstoff Venezianisch Rot, der lange Zeit ein wichtiger Exportartikel war. Das hätte den Drachenbaum fast die Existenz gekostet. 


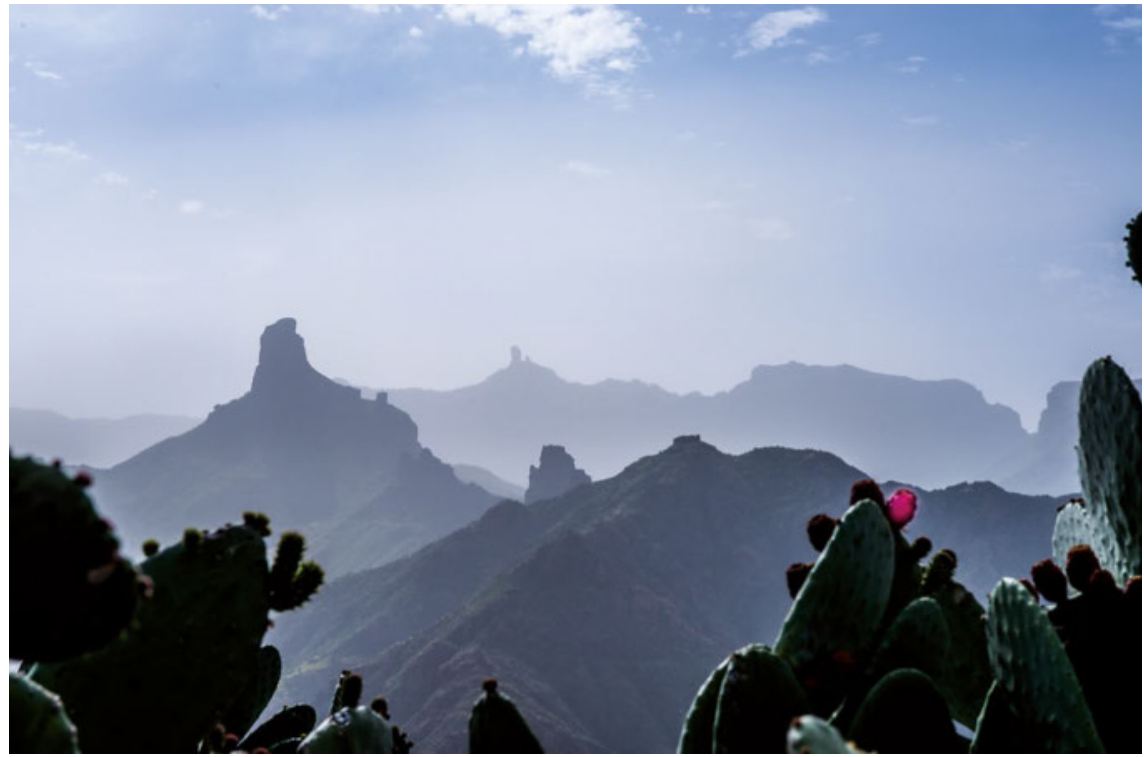

- Abb. 5 Magische Momente trotz vieler ökologischer Probleme. @ Dr. Ferdinand Worm

\section{Monokulturen, Tourismus und der Grundwasserspiegel}

Einen weiteren Höhepunkt für botanisch Interessierte bot die Wanderung durch den Barranco de la Mina, die wasserreichste Schlucht der Insel mit ihrer üppigen Vegetation. Die Blutrote Cinerarie (Pericallis cruenta (L'Hér.) Bolle.), leuchtend gelbe Hahnenfußarten, Moose, Farne und Flechten, die Berg-Tinguarra (Tinguarra montana (Webb ex Christ) A. Hansen \& G. Kunkel), ein stattlicher Doldenblütler und vieles mehr. Der Barranco de la Mina war übrigens die einzige Schlucht, in der wir fließendes Wasser fanden. Sie vermittelte uns einen Eindruck von dem, was Besucher früherer Jahrhunderte auf Gran Canaria überall vorgefunden haben müssen: Leonardo
Torriani berichtete 1590 in seiner Schrift Die Kanarischen Inseln und ihre Urbewohner von „...einer Unzahl ausgezeichneter Quellen, welche die Zahl von 5000 erreichen“. George Glass schrieb 1777 in seiner Geschichte der Entdeckung und Eroberung der Kanarischen Inseln: „Die Insel ist sehr wasserreich und hat einen Überfluss an Holz von verschiedener Art.“ Noch 1815 schreibt der Geologe Leopold von Buch in seinem Werk Physicalische Beschreibung der canarischen Inseln: „Wasser läuft überall“.

Das hat sich drastisch geändert. Wald ist nur noch rudimentär vorhanden. Für die Gewinnung des Süßwassers aus den im Norden der Insel vom Meer aufsteigenden Nebeln ist er jedoch unverzichtbar. Bemühungen zur Wiederaufforstung gestalten sich schwierig, denn seit Jahren liegen die Niederschläge unter dem langfristigen Durchschnitt. Süßwasser ist durch die Aktivitäten des Menschen knapp geworden auf Gran Canaria. Seit über 500 Jahren hat die Insel wechselnde landwirtschaftliche Monokulturen $\mathrm{zu}$ verkraften: Zuckerrohr, Weinbau, Bananen und zurzeit insbesondere Tomaten. Der Boden ist erodiert, der Grundwasserspiegel in wichtigen küstennahen Gebieten allein zwischen 1980 und 2000 um bis zu $25 \mathrm{~m}$ gesunken. Salzwasser drückt seitdem in die Süßwasservorräte unter der Insel nach und verschlechtert deren Qualität. Zur Süßwassergewinnung ist man auf elektro-osmotische Meerwasserentsalzung angewiesen. Größter Wasserverbraucher ist mit ca. $80 \%$ der gesammelten natürlichen Niederschläge die küstennahe Landwirtschaft auf der Insel, gefolgt vom Tourismus.

Bleibt zu hoffen, dass man auf Gran Canaria zu umweltverträglichen Anbaumethoden und Tourismusformen findet und die Lebensgrundlage für die artenreiche Pflanzenwelt und damit für Mensch und Tier nicht weiter zerstört ( $\triangleright$ Abb. 5). Viele Pflanzen des sogenannten Sukkulentenbuschs der Insel sind auf Wasserknappheit eingestellt und kommen mit erstaunlich wenig Niederschlägen aus. Um aber auch im Landesinneren die unzähligen Felder und Gärten auf den von den Vorfahren angelegten Terrassen weiter bestellen und dort leben zu können, braucht es Wasser.

Für den AK-Phyto

Cäcilia Brendieck-Worm 